\title{
Receptor response and soma leakiness in a simulated spiking neural controller for a robot
}

\author{
David Bowes, Rod Adams, Lola Cañamero, Volker Steuber, Neil Davey \\ Science and Technology Research Institute \\ University of Hertfordshire \\ College Lane \\ Hatfield,Hertfordhsire \\ United Kingdom \\ \{D.H.Bowes, R.G.Adams, L.Canamero, V.Steuber, N.Davey \\ aherts.ac.uk
}

\begin{abstract}
This paper investigates different models of leakiness for the soma of a simulated spiking neural controller for a robot exhibiting negative photo-taxis. It also investigates two models of receptor response to stimulus levels. The results show that exponential decay of ions across the soma and of a receptor response function where intensity is proportional to intensity is the best combination for dark seeking behaviour.
\end{abstract}

\section{Introduction}

In real neural systems it is known that leakiness in individual neurons can be caused by a variety of physical processes which can in turn lead to a variety of temporal profiles. Moreover the response of receptors to intensity of stimulus could be either linear or non linear [Kandel et al 2000]. Although different mechanisms of leakiness are possible, some are easier to implement in a robot than others. It is important to identify which mechanisms will require the least computational effort while producing the desired behaviour.

In this paper we investigate the effect of the specific function representing leakiness and receptor response, to the ability of an artificial neural system to respond appropriately to stimulus gradients in order to perform negative photo-taxis.

Photo-taxic robots [Bisset and Vandenberg 1997, Floreano 2005, French and Canamero 2005] based on Braitenberg vehicles [Braitenberg 1984], controlled by artificial neural networks, have produced both varying results and success [Bisset and Vandenberg 1997]. We have developed a robot controller using a simplified artificial spiking neuron model, implemented in an event driven simulation [Rochel and Martinez 2003, Bowes et al 2007]. The artificial neuron has a leaky soma which links to an axon hillock. The axon hillock initiates spikes in one or more collaterals attached to the axon hillock. See Figure 1 for an illustration of the artificial spiking neuron. 


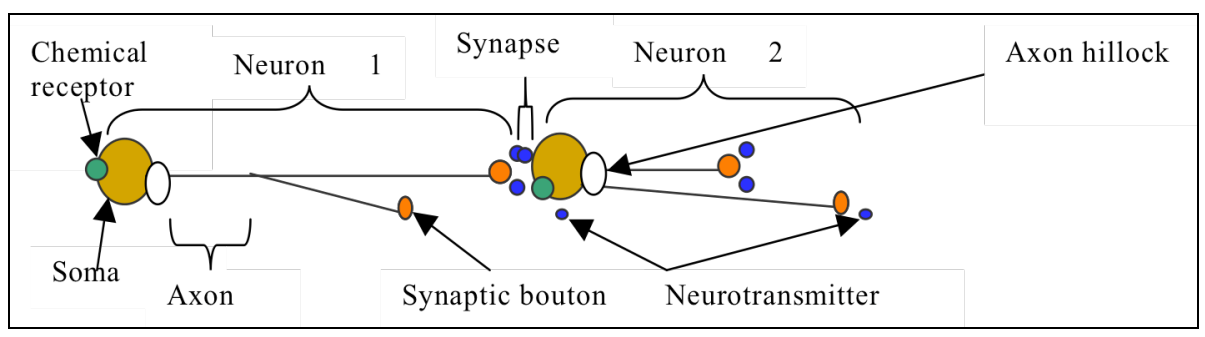

Fig. 1: The model of a simplified spiking neuron showing soma, axons, chemical receptor, synapse and neurotransmitter (note that the dendritic tree is not modeled).

Using a modified Braitenberg fear vehicle [Braitenberg 1984], we simulated the behaviour of negative photo-taxis. In the original Braitenberg fear vehicle the output of the left light sensor is used to set the power output on the left motor, and a corresponding arrangement is made on the right side of the vehicle. In our modified arrangement we add sensory neurons, connected to interneurons, which then connect to the motor. This gives a potentially wider range of behaviours. However this arrangement was only satisfactory when the light intensity difference between the two light sensors was large. When the differences were not large, the robot was unable to correctly orientate itself towards darkness.

Two aspects of the model that could account for this lack of sensitivity were thought to be: the nature of the leakiness of the soma and the relationship between light intensity and firing rate of the light receptors.

In real neurons leakiness is controlled by a variety of mechanisms including voltage dependent and independent ion channels, active transport though pumps, exchanges and axial currents [Kandel et al 2000].

Subsequently we studied two models for leakiness: linear decay in voltage, which simple, and an exponential decay which is more plausible and could be caused by ions passing through pores along a concentration gradient, see Figure 2. 

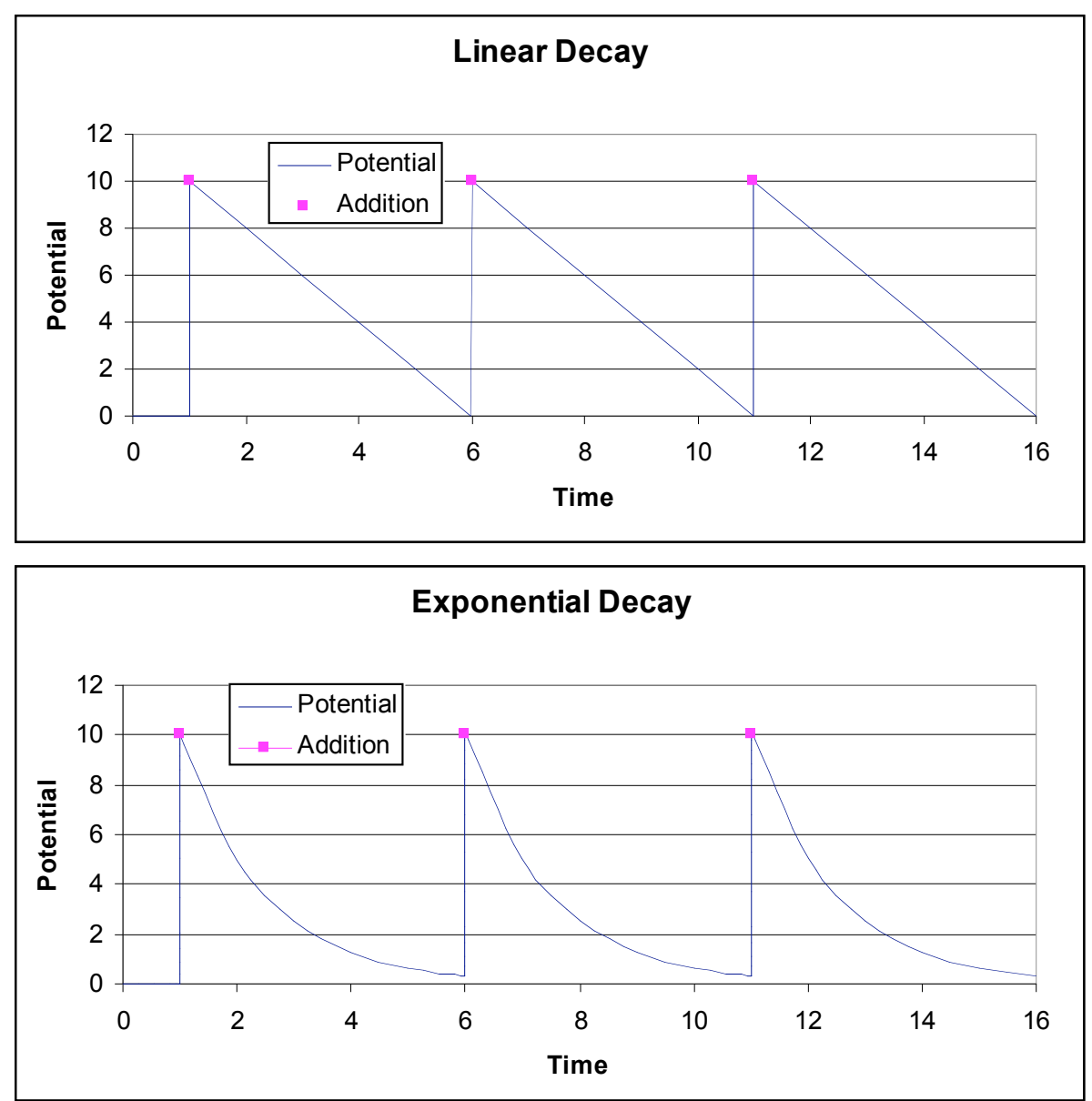

Fig. 2. Membrane potential with different decay functions with a constant rate of voltage additions generated by the arrival of spike events (designated by Addition points in the graph).

Both of these are biologically possible, but the latter system, which does not involve the direct use of energy, is more likely in a biological organism where parsimony of design is often found.

The relationship between light intensity and input current to a receptor neuron is complex and may lead to a variety of mappings between light intensity and the firing rate of a receptor.

To investigate this, two models for firing rate of a receptor cell, under different light intensities, were studied: inter spike time being inversely proportional to light intensity, or, inter spike time being proportional to the difference between the maximum possible intensity and actual intensity. The first of these produces a linear relationship between light intensity and firing rate and the second produces a hyperbolic relationship. Both of these are biologically plausible [Kandel et al 2000]. The first model would result in firing rate being proportional to light intensity and the second method would produce a non-linear relationship as shown in Figure 3. 


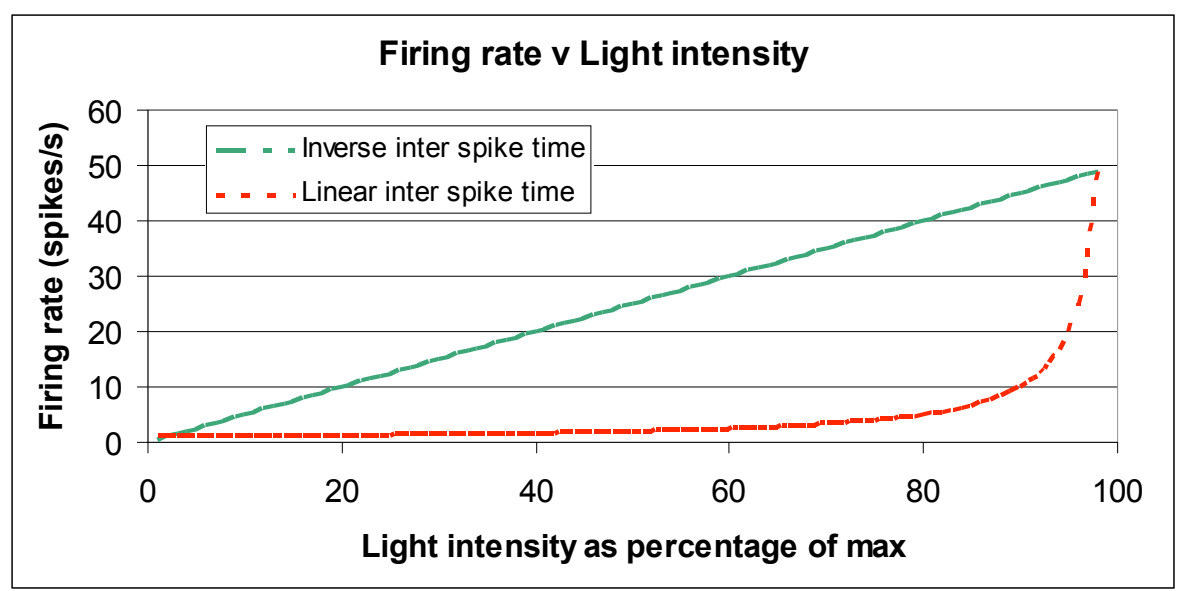

Fig. 3. Firing rates of the sensory neuron using a linear and hyperbolic relation. Note the poor discrimination of the hyperbolic function at low light intensities.

\section{Methodology}

The experiment to test which function produced the greatest rotation was carried out on a robot, simulated in software. A schematic of the robot is shown in Figure 4.

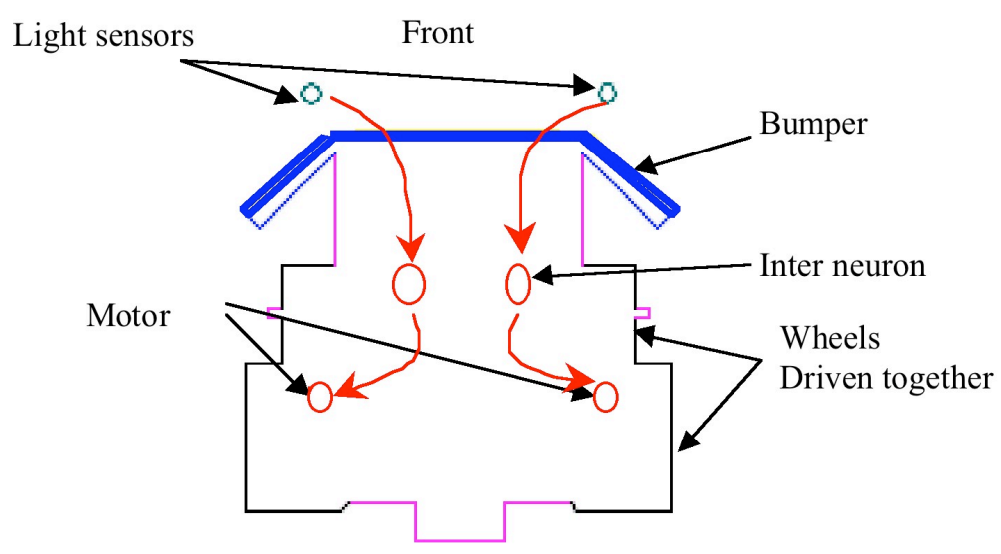

Fig. 4. A schematic of the robot indicating the position of the two light sensors and two interneurons which attach to the motors. Note the connections are excitatory which causes the vehicle to perform negative photo-taxis.

Simulated receptor neurons were attached to light sensors. These sensory neurons then connected to interneurons which subsequently attached to the motors with excita- 
tory ipsilateral connections (left receptor connects to left motor). At the start of each experiment the robot was placed perpendicular to a linear light gradient, see Figure 5.

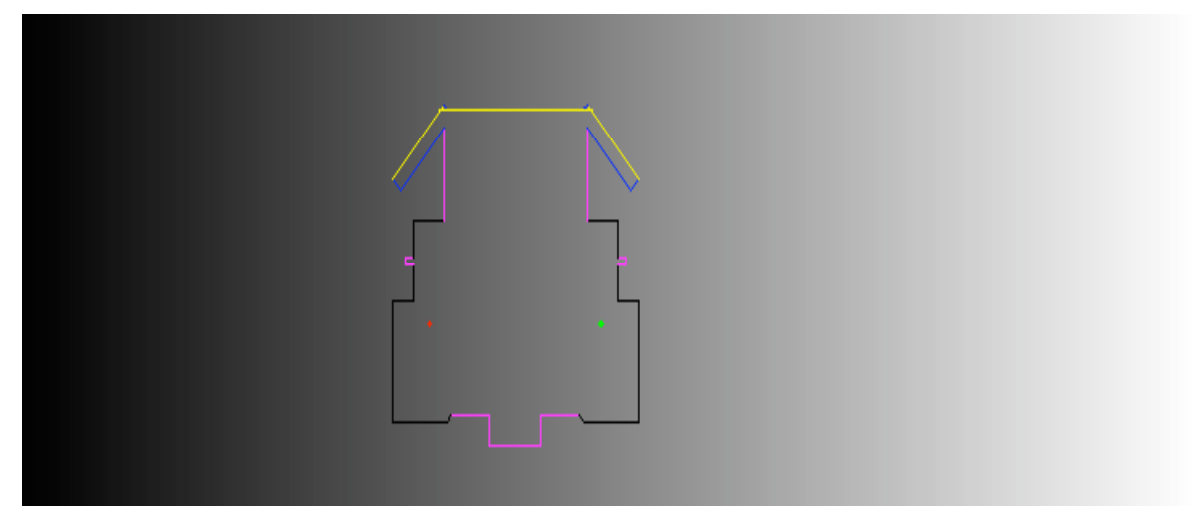

Fig. 5. The Robot on the linear light gradient. The robot is placed orthogonally to the gradient and is "pinned" in position so that it can only rotate (X marks the vertical axis of rotation.

The simulation prevented forward motion of the robot, but allowed rotation so that the robot would spin on the spot (similar to an insect being tested for pheromone orientation in a wind tunnel). The simulation was run for 10 seconds and the total angle of rotation was recorded. The robot was placed at incremental distances from the left side of the gradient, providing 9 data samples. Each permutation of decay model, receptor firing model and position was repeated 7 times, resulting in a total of 252 experiments.

The two models for potential decay in the soma of the interneuron are:

- Linear decay: which is very simple to calculate

$$
c_{(t+1)}=c_{t}-\alpha
$$

- Exponential decay: biologically more plausible

$$
c_{(t+1)}=c_{t} \beta
$$

Where $c$ is the synaptic concentration

with constants $\alpha$ and $\beta$, where $\beta<1$.

Two models for determining the time between firings of light receptor neurons were:

- Inverse:

so

$$
\begin{aligned}
& T \propto \frac{1}{i}+T_{\min } \\
& F \propto i
\end{aligned}
$$

producing a linear relationship between light intensity and neuron firing rate.

- Linear: 


$$
\begin{aligned}
\quad T & \propto(k-i)+T_{\text {min }} \\
\text { so } \quad F & \propto \frac{1}{k-i}
\end{aligned}
$$

producing a hyperbolic relationship between light intensity and firing rate.

Where $k$ is the maximum stimulus level achievable, $i$ is the intensity of stimulation $F$ is the firing rate and $T_{\min }$ is a constant which prevents the period being less than $0.01 \mathrm{~s}$ (below this value the amount of processing increases significantly).

\section{Results}

The full results for all four experimental conditions are given in Figure 6. This shows that significant rotation only occurs under the condition of exponential voltage decay and inverse inter spike time and then only in the dark region.

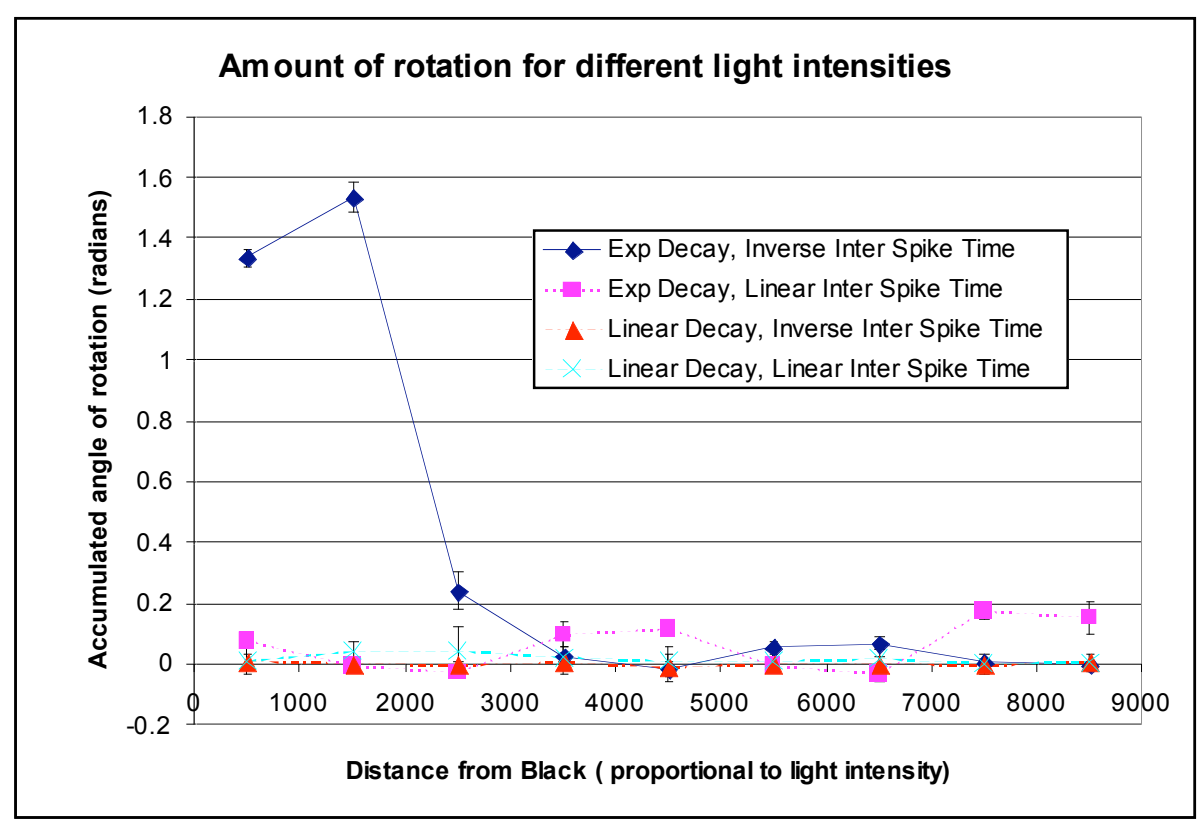

Fig. 6. A graph of the amount of rotation achieved. This shows the results for the four conditions: exponential voltage decay and inverse inter spike time, exponential voltage decay and linear inter spike time, linear voltage decay and inverse inter spike time, and linear decay and linear inter spike time. The exponential voltage decay and inverse inter spike time is the only configuration where significant rotation occurred.

A study of the motor logs for all conditions indicated that at high light levels, both motors had been turned on to the maximum level due to very high rates of input firings. This had occurred because the soma of the interneuron had saturated and had prevented the voltage dropping below the threshold value for spiking. 
Linear decay of potential performed particularly poorly. In high light intensity the decay of voltage in the interneuron was not sufficient to allow the voltage to fall below the firing threshold - the rate of decay in this model is constant and is therefore not related to the actual voltage. Moreover at low light intensities the voltage decay was too rapid to allow the voltage to ever exceed the firing threshold. Exponential decay of potential was much better: in this case the rate of decay is proportional to voltage, producing much greater sensitivity at the interneuron.

As already noted a linear relationship between inter spike times and light intensity at the light receptor neurons gives rise to a hyperbolic relationship between intensity and firing rate. This gives poor discrimination at low light levels and the results demonstrate the inadequacy of this encoding.

\section{Discussion}

In real neural systems it is known that leakiness in individual neurons can be caused by a variety of physical processes, which can lead to a variety of temporal voltage profiles. Moreover the response of receptors to intensity of stimulus could be either linear or non linear [Kandel et al 2000].

Our results show that for the example of a negatively photo-taxic robot, an exponential decay of membrane potential and an inverse relationship between light intensity and inter spike time (resulting in a linear relationship between light intensity and firing rate) produces the greatest rotation at low light levels.

At high light levels the rotation is limited due to both motors being stimulated at the maximum rate because the interneurons receive levels of stimulus which prevents the membrane potential from dropping below threshold.

Interestingly, the most effective neural model is also the most biologically plausible. Diffusion of ions through pores would naturally give rise to an exponential decay whereas linearization of the decay would require additional voltage dependant mechanisms.

The modified Braitenberg wiring using interneurons is biologically more realistic than the original vehicle because it involves some level of processing by the interneurons, but it is not sophisticated enough to cope with the full range of light levels. We are currently investigating models of dynamic normalisation to achieve greater sensitivity to small differences in light intensity.

\section{References}

[Hodgkin and Huxley 1952] Hodgkin, A. L. and Huxley, A. F. (1952). A quantitative description of ion currents and its applications to conduction and excitation in nerve membranes. J. Physiol. (Lond.), 117:500-544.

[Bowes et al 2007] Bowes,D.H; Adams,R.G; Canamero,L; Davey,N (2007) "A Simplified Spiking Neural Network for a Robot" Technical report, Science and Technology Research Institute, University of Hertfordshire

[Braitenberg 1984] Braitenberg. Vehicles: experiments in synthetic psychology. 1984. Elliot Sober 
[Floreano 2005] Floreano D Evolutionary Robotics:A Short Tutorial 2005

[French and Canamero 2005] French, R.L.B.; Canamero, L., "Introducing Neuromodulation to a Braitenberg Vehicle," Robotics and Automation, 2005. ICRA 2005. Proceedings of the 2005 IEEE International Conference on , vol., no., pp. 4188-4193, 18-22 April 2005

[Bisset and Bisset 1997] Bisset, D. L.; Vandenbergh, R. C. (1997) The Dynamics of PhotoTaxis: Applying the Agent Environment Interaction System to a Simple Braitenberg Robot European Conference on Artificial Life Vol 4, 327-336

[Kandel et al 2000] Kandel ER, Schwartz JH, Jessell TM 2000. Principles of Neural Science, 4th ed. McGraw-Hill, New York

[Rochel and Martinez 2003] Rochel, O; Martinez, D An event-driven framework for the simulation of networks of spiking neurons, European Symposium in Artificial Neural Networks, 2003 pp 295-300

[Shepherd 2004] GM Sheperd 2004. The Synaptic Organization of the Brain ,5thRev, Oxford UP ,New York.

1. Baldonado, M., Chang, C.-C.K., Gravano, L., Paepcke, A.: The Stanford Digital Library Metadata Architecture. Int. J. Digit. Libr. 1 (1997) 108-121 